\title{
Loss of TNR causes a nonprogressive neurodevelopmental disorder with spasticity and transient opisthotonus
}

\author{
Matias Wagner, $\mathrm{MD} \mathbb{1 0}^{1,2,3}$, Jonathan Lévy, $\mathrm{MD}, \mathrm{PhD}^{4}$, Sabine Jung-Klawitter, $\mathrm{PhD}^{5}$, \\ Somayeh Bakhtiari, $\mathrm{PhD}^{6,7}$, Fabiola Monteiro, MD, $\mathrm{PhD}^{8}$, Reza Maroofian, $\mathrm{PhD}^{9}$, \\ Tatjana Bierhals, MD ${ }^{10}$, Maja Hempel, MD ${ }^{10}$, Monique Elmaleh-Bergès, MD ${ }^{11}$, Joao P. Kitajima, PhD $^{8}$, \\ Chong A. Kim, MD, PhD ${ }^{12}$, Julia G. Salomao, MD ${ }^{12}$, David J. Amor, MBBS, PhD ${ }^{13,14}$, \\ Monica S. Cooper, MBBS ${ }^{13,14}$, Laurence Perrin, MD ${ }^{4}$, Eva Pipiras, PhD ${ }^{15}$, Axel Neu, MD ${ }^{16}$, \\ Mohammad Doosti, PhD ${ }^{17}$, Ehsan G. Karimiani, MD, PhD ${ }^{18}$, Mehran B. Toosi, MD ${ }^{19}$, \\ Henry Houlden, MD, $\mathrm{PhD}^{9}$, Sheng Chih Jin, $\mathrm{PhD}^{20}$, Yue C. Si, MD, PhD ${ }^{21}$, Lance H. Rodan, MD ${ }^{22}$, \\ Hanka Venselaar, PhD ${ }^{23}$, Michael C. Kruer, MD ${ }^{6,7}$, Fernando Kok, MD, PhD ${ }^{8}$, Georg F. Hoffmann, MD ${ }^{5}$, \\ Tim M. Strom, MD ${ }^{1}$, Saskia B. Wortmann, MD, PhD ${ }^{1,2,24}$, Anne-Claude Tabet, MD, PhD ${ }^{4,25}$ and \\ Thomas Opladen, MD ${ }^{5}$
}

\begin{abstract}
Purpose: TNR, encoding Tenascin-R, is an extracellular matrix glycoprotein involved in neurite outgrowth and neural cell adhesion, proliferation and migration, axonal guidance, myelination, and synaptic plasticity. Tenascin- $\mathrm{R}$ is exclusively expressed in the central nervous system with highest expression after birth. The protein is crucial in the formation of perineuronal nets that ensheath interneurons. However, the role of Tenascin- $\mathrm{R}$ in human pathology is largely unknown. We aimed to establish TNR as a human disease gene and unravel the associated clinical spectrum.
\end{abstract}

Methods: Exome sequencing and an online matchmaking tool were used to identify patients with biallelic variants in TNR.

Results: We identified 13 individuals from 8 unrelated families with biallelic variants in TNR sharing a phenotype consisting of spastic para- or tetraparesis, axial muscular hypotonia, developmental delay, and transient opisthotonus. Four homozygous lossof-function and four different missense variants were identified.

Conclusion: We establish TNR as a disease gene for an autosomal recessive nonprogressive neurodevelopmental disorder with spasticity and transient opisthotonus and highlight the role of central nervous system extracellular matrix proteins in the pathogenicity of spastic disorders.

Genetics in Medicine (2020) 22:1061-1068; https://doi.org/10.1038/ s41436-020-0768-7

Keywords: TNR; spastic tetraparesis; cerebral palsy; exome sequencing; developmental disorder

\section{INTRODUCTION}

Next-generation sequencing (NGS) methods have revolutionized the diagnostic opportunities of neurogenetic diseases. ${ }^{1,2}$ While in the past disease entities were primarily described based on specific clinical findings, the rapidly evolving sequencing technologies have added a new dimension of entities based on genetic diagnoses. A combination of both has characterized syndromes such as hereditary spastic paraparesis ${ }^{3}$ and dystonia. ${ }^{4}$ However, genetic diagnostics in diseases that do not fit into any of the established categories is difficult and often requires interdisciplinary approaches and the inclusion into research projects.

${ }^{1}$ Institute of Human Genetics, Faculty of Medicine, Technical University München, Munich, Germany; ${ }^{2}$ Institute of Human Genetics, Helmholtz Zentrum München, Deutsches Forschungszentrum für Gesundheit und Umwelt $(\mathrm{GmbH})$, Neuherberg, Germany; ${ }^{3}$ Institut für Neurogenomik, Helmholtz Zentrum München, Deutsches Forschungszentrum für Gesundheit und Umwelt (GmbH), Neuherberg, Germany; ${ }^{4}$ Genetics Department, AP-HP, Robert-Debré University Hospital, Paris, France; ${ }^{5}$ Division of Neuropediatrics and Metabolic Medicine, University Children's Hospital, Heidelberg, Germany; ${ }^{6}$ Barrow Neurological Institute, Phoenix Children's Hospital, Phoenix, AZ, USA; ${ }^{7}$ Departments of Child Health, Neurology, Cellular \& Molecular Medicine and Program in Genetics, University of Arizona College of Medicine, Phoenix, AZ, USA; ${ }^{8}$ Mendelics Genomic Analysis, São Paulo, São Paulo, Brazil; ${ }^{9}$ Department of Neuromuscular Disorders, UCL Queen Square Institute of Neurology, London, UK; ${ }^{10}$ Institute of Human Genetics, University Medical Center Hamburg-Eppendorf, Hamburg, Germany; ${ }^{11}$ Department of Pediatric Radiology, Robert Debré Hospital, AP-HP, Paris, France; ${ }^{12}$ Genetic Unit, Instituto da Criança-HCFMUSP, Universidade de Sao Paulo, Sao Paulo, Brazil; ${ }^{13}$ Murdoch Children's Research Institute, Royal Children's Hospital, Melbourne, Melbourne, VIC, Australia; ${ }^{14}$ Department of Paediatrics, University of Melbourne, Melbourne, VIC, Australia; ${ }^{15}$ Department of Cytogenetics, Jean-Verdier Hospital, Paris 13 University, Embryology and Histology, AP-HP, Bondy, France; ${ }^{16}$ Department of Pediatrics, University Medical Center Hamburg-Eppendorf, Hamburg, Germany; ${ }^{17}$ Department of Genetics, Next Generation Genetic Polyclinic, Mashhad, Iran; ${ }^{18}$ Genetics Research Centre, Molecular and Clinical Sciences Institute, St. George's, University, London, UK; ${ }^{19}$ Department of Pediatric Neurology, Ghaem Hospital, Mashhad University of Medical Sciences, Mashhad, Iran; ${ }^{20}$ Laboratory of Human Genetics and Genomics, The Rockefeller University, New York, NY, USA; ${ }^{21}$ GeneDx, Gaithersburg, MD, USA; ${ }^{22}$ Division of Genetics and Genomics, Boston Children's Hospital, Boston, MA, USA; ${ }^{23}$ Centre for Molecular and Biomolecular Informatics, Radboud University Medical Centre, Nijmegen, The Netherlands; ${ }^{24}$ University Childrens Hospital, Paracelsus Medical University, Salzburg, Austria; ${ }^{25}$ Neuroscience Department, Human Genetics and Cognitive Function Unit, Pasteur Institute, Paris, France. Correspondence: Matias Wagner (matias.wagner@mri.tum.de) or Thomas Opladen (Thomas.Opladen@med.uni-heidelberg.de)

These authors contributed equally: Matias Wagner, Jonathan Lévy, Sabine Jung-Klawitter

These authors contributed equally: Anne-Claude Tabet, Thomas Opladen

Submitted 18 November 2019; revised 13 February 2020; accepted: 13 February 2020

Published online: 26 February 2020 
Perineuronal nets constitute a specialized form of the extracellular matrix (ECM) that are composed of the proteogylcans aggrecan, neurocan, and brevican as well as hyaluronan. ${ }^{5}$ TNR encodes Tenascin- $\mathrm{R}$, a member of the tenascin family of ECM glycoproteins, which is crucial for the crosslinking of proteoglycan hyaluronan complexes. ${ }^{6}$ TNR is exclusively expressed in the central nervous system with the exception of a transient expression in Schwann cells during peripheral nerve development. ${ }^{7,8}$ Proteolytic cleavage of the amino-terminal region from the $180-\mathrm{kb}$ protein product gives rise to the smaller $160-\mathrm{kD}$ isoform. ${ }^{9}$ Tenascin- $\mathrm{R}$ is involved in neurite outgrowth, neural cell adhesion, proliferation and migration, fate determination, axonal guidance, myelination, synaptic plasticity, and modulation of sodium channel function. ${ }^{10}$ Tenascin- $\mathrm{R}$ deficient mice are viable and fertile and do not show any obvious disease phenotype. ${ }^{11}$ However, they display increased anxiety and motor coordination impairment in specific tests. ${ }^{12}$ They also present altered synaptic activity with a decrease of extracellular space volume and degree of tortuosity and density of perineuronal nets. ${ }^{13}$ Electrophysiological studies revealed abnormal formation of perineuronal nets and reduced conduction velocity of the optic nerve. ${ }^{11}$ In addition, mice have abnormal hippocampal morphology and reduced coverage of symmetric synapses on pyramidal cells. ${ }^{14}$ During fetal development, $\mathrm{Tnr}^{-/-}$mice display increased numbers of GABAergic interneurons. TNR deficiency has been found to be involved in the regulation of neuronal differentiation at least in the mouse dentate gyrus. ${ }^{15,16}$ The broad variety of findings in the mouse model constitutes TNR as an excellent candidate gene for neurogenetic disease. Indeed, two recent reports have identified cases with biallelic loss-of-function (LoF) variants in TNR. The first patient was born from consanguineous Lebanese parents and presented with intellectual disability and early onset opisthotonic posture (at 4-6 weeks of age); array comparative genomic hybridization identified a homozygous deletion containing all protein coding regions of $T N R$ as well as parts of the $5^{\prime}$-untranslated region of KIAA0040 allowing authors to suggest TNR's implication in brain development and cognition. ${ }^{17}$ The second patient was described in a case series of 100 adults with leukoencephalopathy. Exome sequencing revealed a homozygous LoF variant in TNR (c.1475delG, p.[Arg492Profs*45]) in a Turkish female who developed a floppy head and opisthotonic spasms of her neck and back, generalized dystonia, and spasticity at three months and had mild learning difficulties. ${ }^{18}$

Here, we report 13 patients from 8 unrelated families with biallelic variants in TNR causing a complex syndrome characterized by mild neurodevelopmental delay, axial muscular hypotonia, spasticity, hypokinesia, and transient opisthotonus establishing TNR as a disease gene for spastic para- or tetraparesis.

\section{Patients and samples}

MATERIALS AND METHODS

All patients or their parents gave written informed consent for the pseudonymized clinical data collection, collection and storage of biological samples, experimental analyses, and the publication of relevant findings and images/videos. The study was performed in agreement with the Declaration of Helsinki and approved by the Ethical Committees of the centers participating in this study (Munich, Germany; Hamburg, Germany; Paris, France; Phoenix, AZ, USA; Sao Paulo, Brazil; and London, UK). Percentiles for growth parameters were estimated for all individuals as previously described. ${ }^{19}$ The collaboration was established using the web-based platform GeneMatcher. $^{20}$

\section{Exome sequencing}

Exome sequencing (ES) and TNR Sanger sequencing was carried out independently at five different centers using genomic DNA extracted from leukocytes. Technical details can be found in Supplementary Table 1.

\section{D modeling}

Three-dimensional (3D) modeling was performed using the WHAT IF \& YASARA Twinset with standard parameters. ${ }^{21,22}$ Separate models were created for individual domains using separate Protein Data Bank (PDB) files as templates. Fibronectin domain 1 was modeled on PDB file 3TEU (38\% sequence identity, 105 residues). ${ }^{23}$ Fibronectin domain 9 was modeled on $\mathrm{PDB}$ file $4 \mathrm{U} 3 \mathrm{H}$ ( $31 \%$ sequence identity, 100 residues). ${ }^{24}$ The C-terminal fibrinogen domain was modeled on PDB file 6QNV (61\% sequence identity, 231 residues). Additionally, Fibronectin domain 3 was available as PDB file 1TDQ. ${ }^{25}$ Visualization and subsequent analysis was done using the YASARA \& WHAT IF Twinset.

\section{Patient characteristics}

\section{RESULTS}

Detailed case reports are available in Supplementary Information and are summarized in Table 1 . An extended version of Table 1 can be found in the Supplementary Information. Videos of patients 1, 7, 9, and 10 can be found in the Supplementary Information.

In brief, 13 patients from 8 unrelated families $(7$ males, 6 females, current ages ranging from 1 to 24 years; pedigrees can be found in Fig. 1) were referred for evaluation of developmental delay after uneventful pregnancy, delivery, and postnatal adaptation in most families (see Supplementary case reports). They originated from consanguineous families from Turkey, Morocco, Saudi Arabia, Iraq, and Brazil as well as a nonconsanguineous family from India. No dysmorphic features were noted.

Detailed assessment of patients' history revealed that motor developmental milestones were delayed in all patients: unsupported sitting was achieved at a median age of $12 \pm 9$ months and standing without hold at a median age of $35 \pm 12$ months. Free standing was not achieved at all in four children. Five of $11(45 \%)$ patients showed transient opisthotonic posturing during the first year of life. Cognitive development was mildly or moderately impaired in 6 of 13 patients $(46 \%)$ as judged by the caring physician, however, 


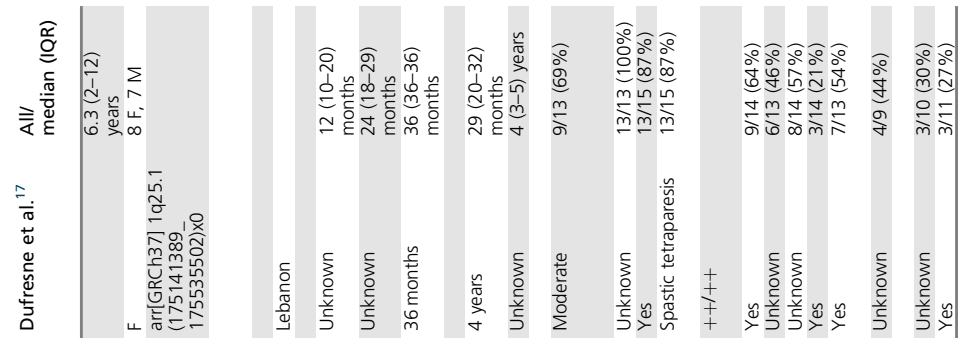

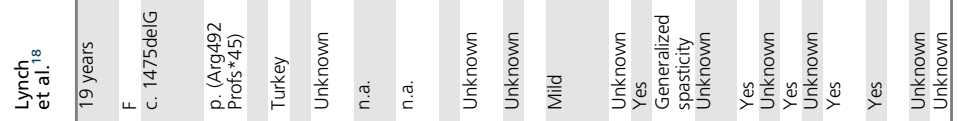

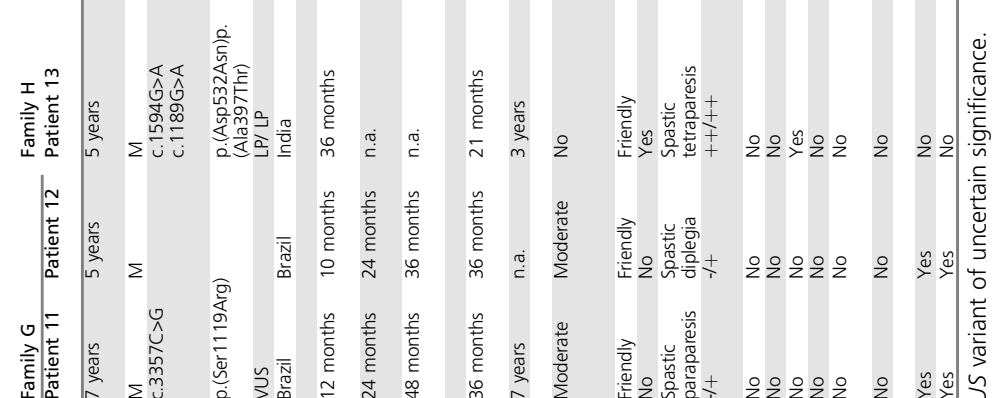

o

冞

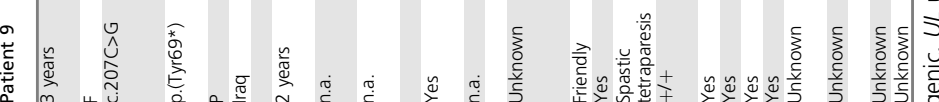

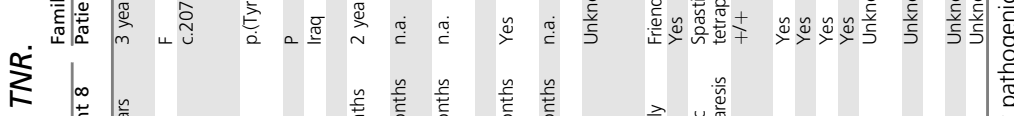

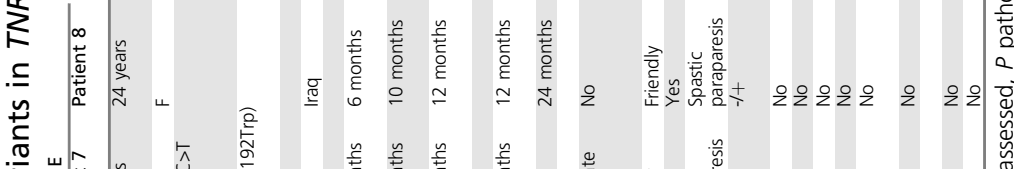

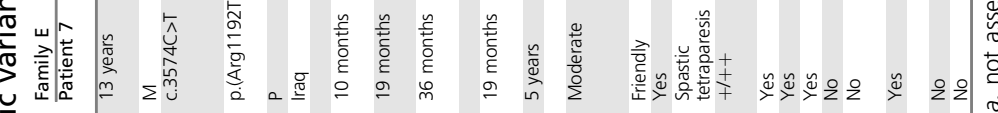

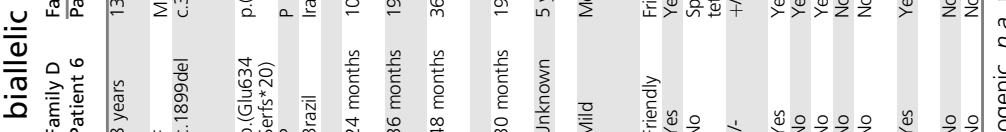

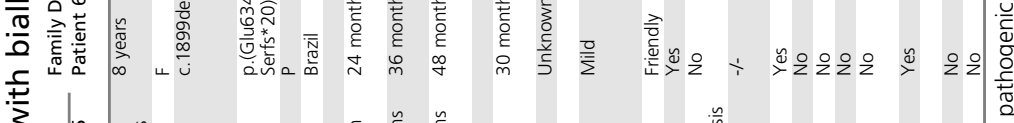

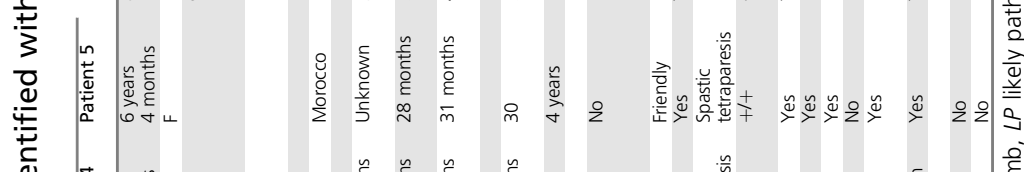

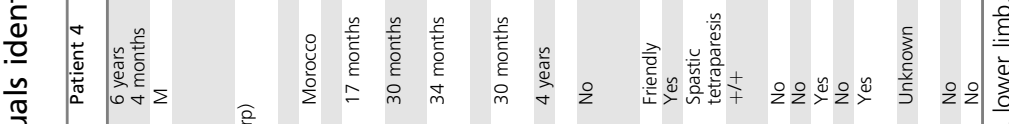

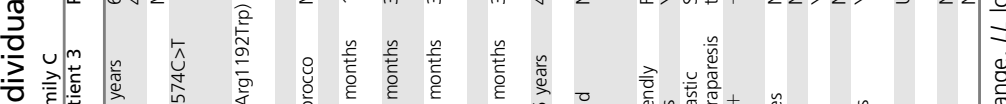

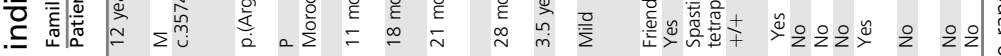

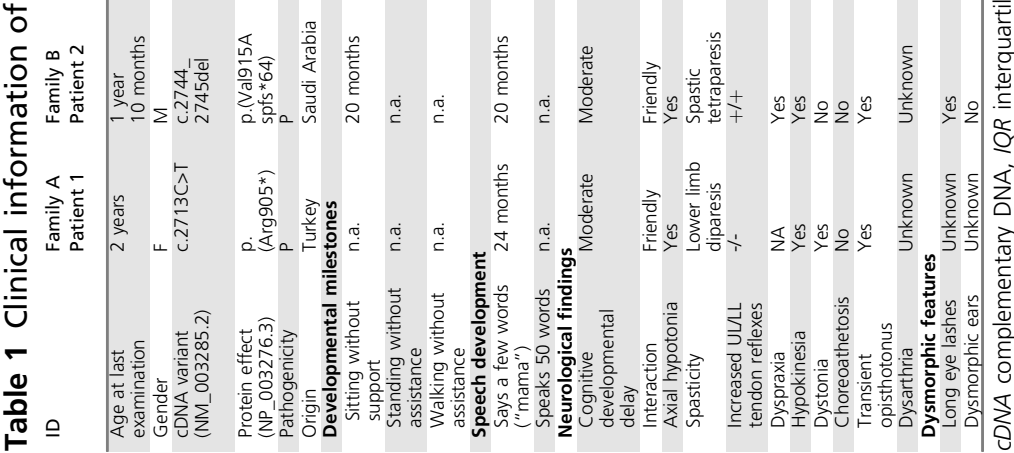


Family A

c. $2713 \mathrm{C}>\mathrm{T}$ p. $\left(\operatorname{Arg} 905^{*}\right)$

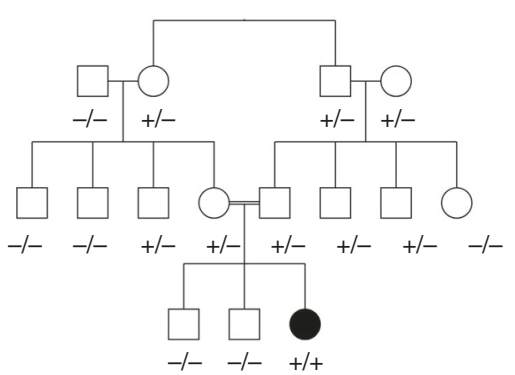

P1
Family B

.2744_2745delTG p.(Val915Aspfs*64)

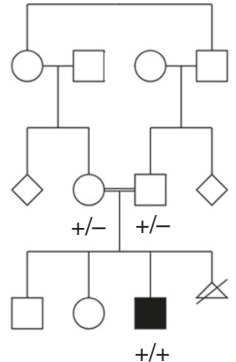

P2
Family C

c.3574C >T p.(Arg1192Trp)

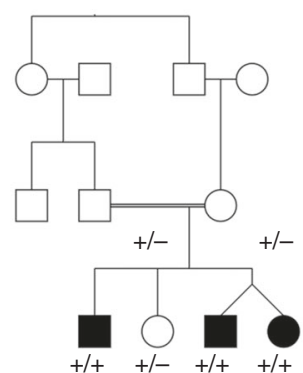

P3

Family $\mathrm{G}$

c.3357C > G p.(Ser1119Arg)
Family D

c.1899delT p.(Glu634Serfs²0)

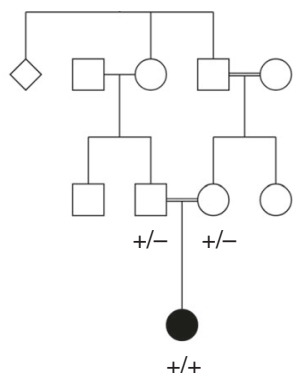

P6
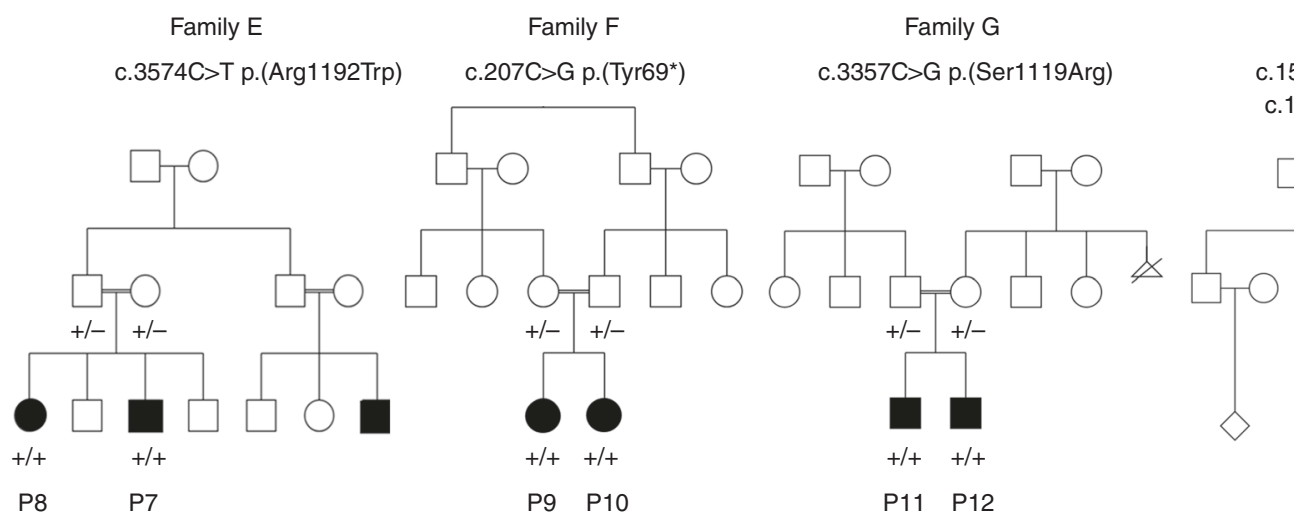

Family $\mathrm{H}$

c.1594G>A p.(Asp532Asn) c.1189G $>$ A p.(Ala397Thr)

Fig. 1 Identification of biallelic TNR variants in eight families. Pedigrees of the families (families A-H) in whom exome sequencing identified biallelic variants in TNR segregating with the disease. Black filled symbols represent affected individuals whereas open symbols represent unaffected family members. $+/+$ indicates that variants were identified in homozygosity; $+/-$ are heterozygous carriers. No variants in TNR were identified in individuals marked with $-1-$.

there is no formal neuropsychological testing available. Four of 11 patients did not have cognitive developmental delay and two cases were too young for a reliable evaluation. Most patients showed delayed language development (12/13; 92\%). However, language impairment was variable, with patient 7 speaking fluently from the age of five years onward, whereas patient 6 still had severe expressive language impairment at the age of eight years, with concomitant dysarthria.

During the assessment of the current neurological status at the median age of 5 years (range 2-24 years) patients typically presented with a spastic tonus dysregulation of two or four extremities (10 affected/12 examined; 83\%) with axial hypotonia $(8 / 12 ; 67 \%)$. Lower limb deep tendon reflexes were normal in two patients and increased in all other examined patients $(10 / 12 ; 83 \%), 7$ patients $(7 / 12 ; 58 \%)$ had increased upper limb reflexes. Notably, all patients were very friendly (happy demeanor) in their interaction with their environment, which was not related to lack of stranger anxiety as observed in children with autism and we did not observe inappropriate laughter. Dyspraxia was noted as a further neurological symptom in 7/13 (54\%) patients. In addition to spastic para- or tetraparesis, also (oromandibular) dystonia (7/13; 54\%), choreoathetosis and parkinsonism could be documented. Cerebellar symptoms were not observed in our patients. The three oldest patients (age $>12$ years) included in our study as well as the case reported ${ }^{18}$ largely caught up the delay in cognitive and motor development after infancy, as reported by the responsible clinicians. Moreover, none of our patients developed additional neurological system involvement over time and the severity of the neurological symptoms did not increase over time. Spasticity neither improved nor deteriorated. This indicates that TNR-associated disease is nonprogressive in nature.

\section{MRI findings in TNR-related disease}

Brain magnetic resonance imaging (MRI) was performed in 11/13 patients. Relevant images or whole MRI data sets have been evaluated by the same pediatric neuroradiologist.

Delayed myelination was observed in four cases: patients 1 , 3,5 , and 9 at the age of 4 months, 1 year 7 months (Fig. 2a), 10 months, and 2 years 8 months (Fig. 2d), respectively. In patient 3 the delayed myelination of the temporal subcortical white matter was persistent at the age of five years (Fig. 2b) compared with a brain MRI of a normal five-year-old boy (Fig. 2c). In contrast, myelination appeared normal on followup at two years in patient 1 , which is in line with the clinical evaluation, where symptom severity remained stable or even showed slight improvements over time.

In two cases, MRI revealed corpus callosum abnormalities: patient 6 had a thin corpus callosum and patient 11 had an 
agenesis of the posterior part of the corpus callosum associated with a splenium agenesis at the age of 10 months (Fig. 1e). Brain MRI was considered to be normal in patients $2,4,7,12$, and 13. Cerebellar abnormalities were not found in any of our cases.

\section{Biallelic variants in $T N R$}

In all index patients, exome sequencing at the local genetic centers did not identify causative variants in established disease genes. Filtering for rare (minor allele frequency [MAF] $<0.5 \%$ in the respective in-house databases) protein altering variants identified biallelic variants in TNR (NM_003285.2) in index patients from all families. Additional potentially biallelic variants identified in the index cases by exome sequencing can be found in the Supplementary Information. Segregation by Sanger sequencing did support TNR as a candidate gene. A web-based collaboration platform, GeneMatcher, ${ }^{20}$ established the collaboration presented here. Pedigrees and the identified variants can be found in Fig. 1.

A total of four LoF and four missense variants were detected. Patient 1 had a homozygous nonsense variant c. $2713 \mathrm{C}>\mathrm{T}$, p.(Arg905*); patient 2 had a homozygous frameshift alteration c.2744_2745delTG; p.(Val915Aspfs*64). The LoFs c.1899delT p.(Glu634Serfs*20) and c.207C>G; p. (Tyr69*) were identified in patient 6 and patients 9 and 10 (family F) respectively.

In the four other families, two homozygous and two compound heterozygous missense variants were identified: in family C (patients $3-5$ ), the variant c. $3574 \mathrm{C}>\mathrm{T}$ is predicted to result in the substitution of a highly conserved C-terminal amino acid position of the fibrinogen-like domain with to date unknown function, p.(Arg1192Trp). Of note, the Cterminal is not affected by the proteolytic cleavage of the 180$\mathrm{kDa}$ TNR protein into the $160-\mathrm{kDa}$ isoform and therefore the variant should be expressed. ${ }^{9}$ The same variant was identified in the index patient of family E (patient 7) and confirmed by Sanger sequencing in the elder sister (patient 8). In the index patient of family $G$ (patient 11), the missense variant c.3357C $>$ G; p.(Ser1119Arg) was found which is located in the C-terminally Fibronectin type III 9 domain, which is also not affected by proteolytic cleavage. The variants c.1189G $>A$ and c.1594G >A (p.[Asp532Asn] and p.[Ala397Thr]) were identified in patient 13 from family $H$ in a compound heterozygous state as determined be segregation analysis using Sanger sequencing. A graphical view of the TNR transcript structure and the protein product as well as the location of the eight novel and one previously published variants can be found in Fig. 3. Variant details including in silico predictions as well as conservation scores can be found in Supplementary Table 2.

All variants detected were not found in a homozygous state in more than 140,000 NGS data sets in the genome aggregation database (gnomAD) ${ }^{26}$ and could not be identified in population-specific databases focused on Middle Eastern ethnicities, i.e., GME variome database (http://igm.ucsd.edu/ gme/ $)^{27}$ and Iranome (http://www.iranome.ir/). ${ }^{28}$

\section{D modeling}

To better understand the pathophysiologic mechanism of missense variants we performed $3 \mathrm{D}$ modeling of the respective variants (Fig. 3b-e). The variant p.(Ala397Thr) is predicted to affect the local structure whereas the variant $\mathrm{p}$. (Asp532Asn) might affect interaction with brevican in the formation of perineuronal nets. The variant p.(Ser1119Arg) is located on the surface of the fibronectin 9 domain most likely impairing interactions made on the surface of this domain. $\mathrm{p}$. (Arg1192Trp) is located in the fibrinogen domain at the Cterminus and is predicted to affect the local structure.

\section{DISCUSSION}

We report biallelic variants in TNR encoding the ECM protein Tenascin- $\mathrm{R}$ in 13 affected individuals from 8 families. The phenotype encompasses a complex neurological disorder characterized by developmental delay with spastic para- or quadriparesis, axial muscular hypotonia, hypokinesia, and transient opisthotonus combined with a happy demeanor and habitus. Moreover, less frequently observed were language problems. Movement disorders (dystonia, parkinsonism) indicate affection of the extrapyramidal motor system in TNR-related disease. While the cardinal clinical features did not differ significantly between the eight families, the grades of phenotypic severity varied to a greater extent. These differences might be explained by the excess of homozygous variants in our consanguineous families (rare biallelic variants can be found in the Supplementary Information).

Brain MRIs revealed cerebral abnormalities in 6 of 11 patients for whom MRIs were available (65\%). This included delayed myelination in four and corpus callosum abnormalities in two cases. The cerebellum was normal in all cases. Given that delayed myelination and corpus callosum hypoplasia are rather unspecific findings, we could not establish a consistently recognizable MRI phenotype. Intrafamilial heterogeneity as assessed in families $C$ and $G$ was considerable with individuals having both normal and pathologic MRIs at comparable ages within individual pedigrees.

The observations, i.e., that none of our patients developed novel neurological system involvement over time and that the MRI findings improved in follow-up investigations, argue against a neurodegenerative aspect and indicate a nonprogressive nature of the disorder.

It is difficult to delimit $T N R$-associated disease from complicated hereditary spastic paraplegia (HSP), which is characterized by progressive lower limb spasticity and weakness in combination with additional neurologic features such as cognitive deficits, movement disorder, or neuropathy. However, based on the core phenotype, i.e., the spastic affection of both upper and lower limbs as well as this nonprogressive nature of the symptoms, we regard TNRrelated disease as different from HSP.

So far, only two homozygous variants - a frameshift variant, c.1475delG, p.(Arg492Profs*45), and a homozygous 1q25.1 deletion including $T N R$ - have been reported, suggesting $T N R$ 
a

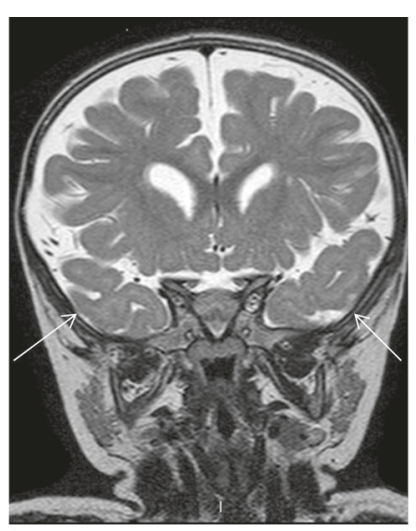

b

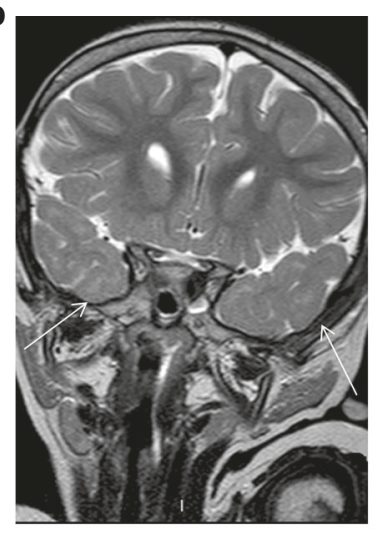

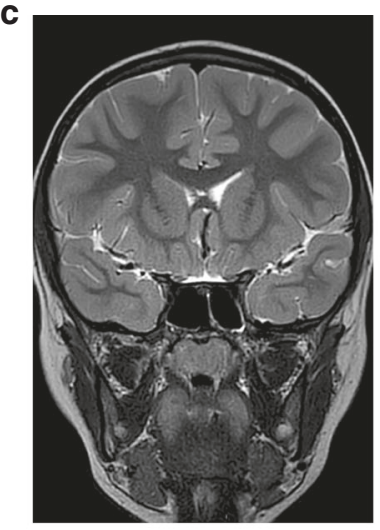

d

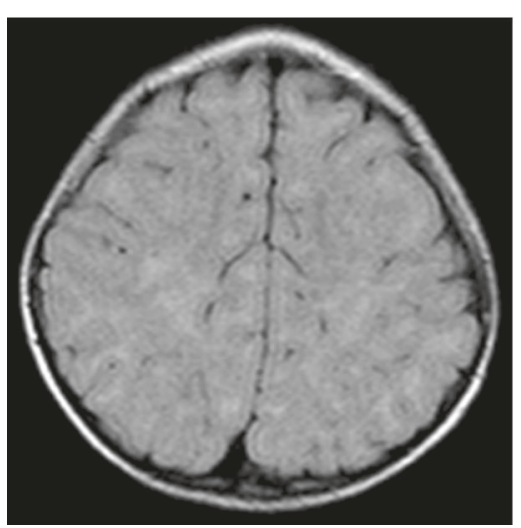

e

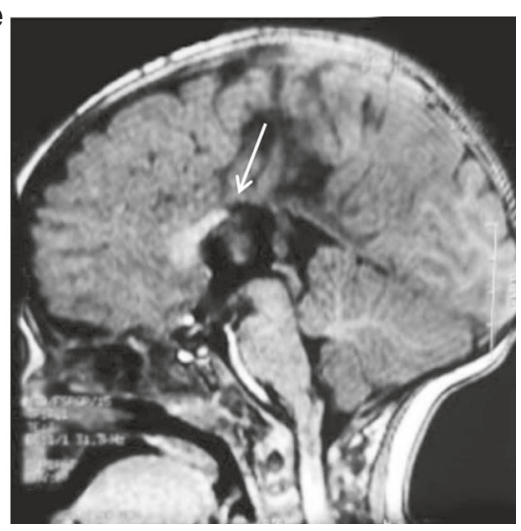

Fig. 2 Magnetic resonance image (MRI) findings in TNR-related neurodevelopmental disease. (a) and (b) Coronal T2-weighted cerebral MRI images of patient 3 at the age of 1 year and 7 months (a) and a follow-up at 5 years (b). (a) Temporal subcortical white matter is not yet myelinated at 1 year 7 months (thin white arrows). (b) MRI revealed a persistent white matter T2 hypersignal at 5 years showing that myelination of the temporal region was not achieved (thin white arrows). (c) Healthy control MRI that was done at age 5 years showing complete myelination of the temporal region. (d) Axial fluidattenuated inversion recovery (FLAIR) weighted sequence of patient 9 at 2 years 8 months, showing a subcortical white matter hypersignal consistent with uncompleted myelination. (e) T1-weighted median sagittal MRI section of patient 11 showing a partial posterior corpus callosum agenesis (large white arrow).

as a candidate gene. Both variants were associated with a neurological phenotype that included spastic quadriparesis, intermittent opisthotonic spasms in the first months of life, delayed motor milestones, dystonia, axial hypotonia, and hyperreflexia, thus resembling the phenotype of our study population (Table 1). ${ }^{17,18}$ Heterozygous LoF variants in TNR are rare (40 in $>280,000$ alleles) and homozygous variants absent are in gnom $A D$, indicating that biallelic pathogenic variants in TNR are likely to cause disease. ${ }^{26}$

We identified four different LoF variants (nonsense and frameshift) in four families as well as four missense variantsc.1189G $>$ A; p.(Ala397Thr), c.1594G $>A$; p.(Asp532Asn), and c.3357C $>$ G; p.(Ser1119Arg) as well as c.3574C $>\mathrm{T}$; p.(Arg1192Trp) - that we identified in two unrelated families. The latter was found in a family from Iraq and Morocco and is absent from control databases. The missense variants are located in the C-terminal fibrinogen-like domain and the fibronectin type III 9 domain, a domain with unknown function to date. There was no significant clinical difference between individuals with LoF and missense variants that would imply a genotype-phenotype correlation indicating a LoF character of the missense variants.
These findings define a novel form of a neurodevelopmental disease presenting with early onset nonprogressive spasticity and developmental delay.

Interestingly, the patient reported by Lynch et al. ${ }^{18}$ carrying the variant c.1475delG, p.(Arg492Profs*45) had a similar phenotype as the individuals identified in our study, but was diagnosed with multiple sclerosis (MS) at the age of 19 due to several T2/FLAIR periventricular white matter, brainstem and cerebellum hyperintensities, and the presence of oligoclonal bands in cerebrospinal fluid (CSF). We believe that, since none of the individuals reported in the present study had MSlike MRI or CSF findings, the published patient potentially was suffering from both TNR-associated neurodevelopmental disease and concomitant MS and that the oligoclonal bands were not associated with the variant in TNR.

TNR is highly expressed in oligodendrocyte precursors with a decreased expression during oligodendrocyte differentiation, suggesting a functional role during myelination. ${ }^{29}$ This might explain why $40 \%$ of our cases had delayed myelination as abnormal MRI findings. TNR is also expressed in type 2 astrocytes and few neuronal cells in the spinal cord, retina, cerebellum, and hippocampus, especially in perineuronal nets 


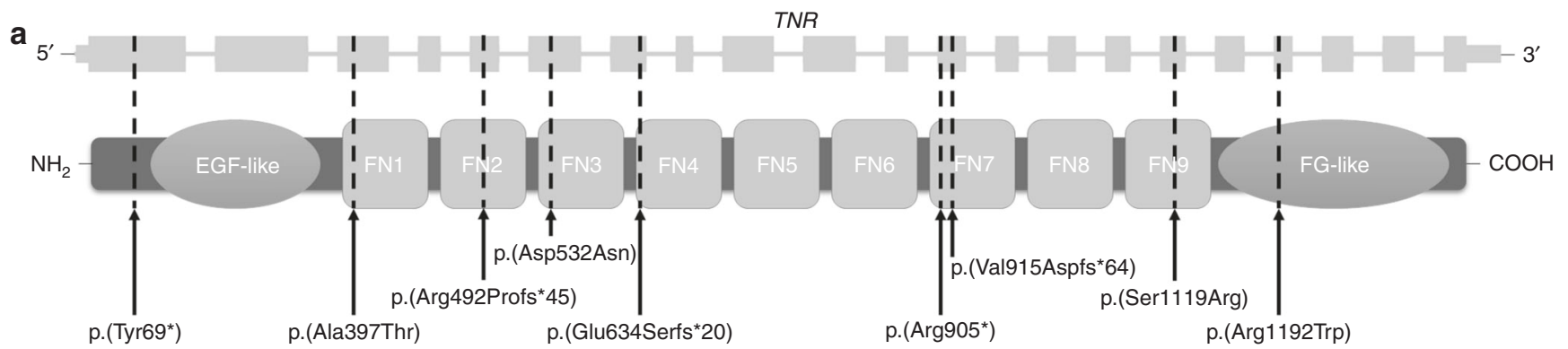

b

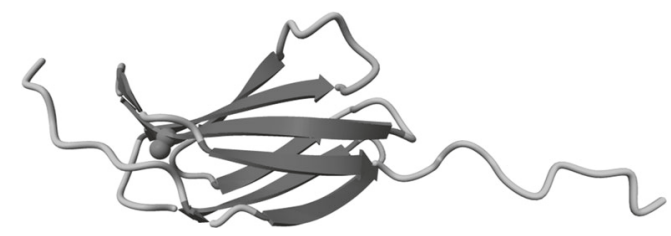

d

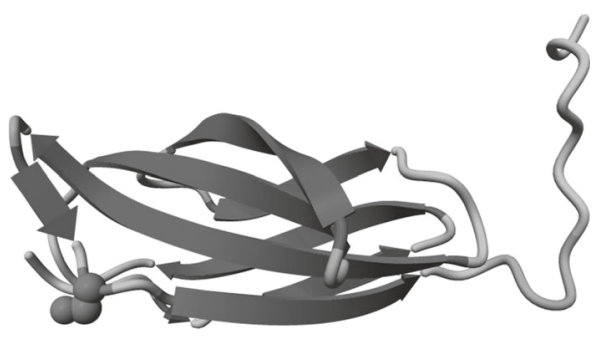

C

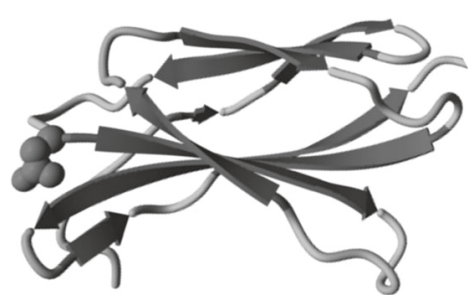

e

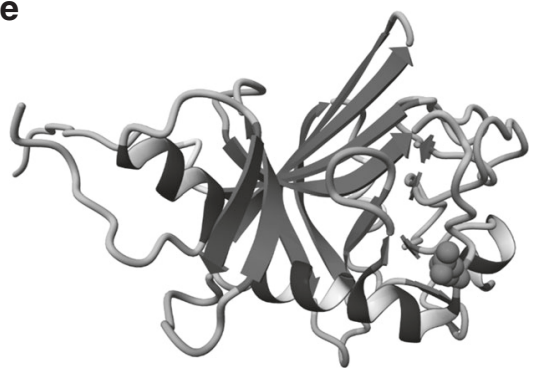

Fig. 3 Graphical view of the location of the eight variants identified with respect to transcript structure and protein product as well as 3D modeling of missense variants. (a) TNR encodes a 21-exon transcript (NM_003285.2). The protein encompasses an N-terminal epidermal growth factor-like domain depicted in orange, nine fibronectin type III domains displayed as blue boxes, and a C-terminal Fibrinogen-like domain colored in green. The arrows and dotted lines mark the positions of the published and novel variants. (b-e) 3D modeling of the missense variants. The mutant amino acid is displayed in magenta, $\alpha$-helices are depicted in blue, $\beta$-strands in red, and loops in cyan. (b) The variant p.(Ala397Thr) is located in fibronectin domain 1 , where the mutated alanine is buried in the core. Mutation of this small and hydrophobic residue into a slightly larger one with an extra hydrophilic sidechain is predicted to affect the local structure. (c) The variant p.(Asp532Asn) occurs on the surface of fibronectin domain 3 . The sidechains of both amino acids have the same size and both are hydrophilic. However, aspartate is negatively charged whereas asparagine is neutral. The variant might affect interaction with brevican in the formation of perineuronal nets. (d) The variant p.(Ser1119Arg) occurs on the surface of fibronectin domain 9. However, whereas serine is small and neutral, arginine is large and positively charged. This could affect any interactions made on the surface of this domain. (e) The variant $p$. (Arg1192Trp) is located in the fibrinogen domain at the C-terminus. The arginine at this position is necessary for hydrogen bonds that stabilize the structure. Mutation into the large and hydrophobic sidechain of tryptophan is predicted to affect the local structure.

surrounding inhibitory interneurons. ${ }^{30}$ As tenascins are a crucial, integral part of perineuronal nets and are involved in the maturation and maintenance of neuronal networks, ${ }^{31}$ a disruption of synaptic plasticity could explain the neurodevelopmental phenotype in our patients. The spatiotemporal expression pattern with the highest expression of TNR in the developing brain is in line with the observed nonprogressive nature of the disease. Even though the behavioral phenotype of TNR-deficient mice does not resemble the symptoms observed in our patients, the mouse model clearly indicates that loss of TNR results in a neurodevelopmental defect.

To date, perineuronal nets or central nervous system ECM proteins have not been implicated in the pathogenesis of spastic disorders, but their dysfunction or loss has been observed in Alzheimer disease, fragile X syndrome, and schizophrenia. ${ }^{32}$ In addition, TNR has been described as a candidate risk gene for Parkinson disease ${ }^{33}$ and a recent genome-wide association study has linked the locus encompassing TNR with attention deficit-hyperactivity disorder. ${ }^{34}$

The identification of novel disease genes often reveals disease-associated molecular mechanisms. Establishing TNR as a disease gene is the first link of central nervous system ECM proteins and perineuronal nets to the pathogenesis of neurodevelopmental disorders.

In conclusion, TNR deficiency causes an early onset and nonprogressive neurodevelopmental disorder characterized by axial hypotonia, spasticity, developmental delay, hypokinesia, and transient opisthotonus.

\section{SUPPLEMENTARY INFORMATION}

The online version of this article (https://doi.org/10.1038/s41436020-0768-7) contains supplementary material, which is available to authorized users. 


\section{DATA AVAILABILITY}

The authors declare that the data supporting the findings of this study are available within the article and its supplementary material. Raw sequencing data are available from the corresponding authors on request if in line with the provided consent of the families.

Variants have been submitted to ClinVar (https://www.ncbi.nlm. nih.gov/clinvar/):

c.3574C>T, p.(Arg1192Trp): Accession ID: VCV000691953

c.3357C>G, p.(Ser1119Arg): VCV000691956

c.2742_2743del, p.(Val915fs): VCV000691952

c.2713C>T, p.(Arg905Ter): VCV000691951

c.1899del, p.(Glu634Serfs*20): VCV000691954

c.1594G>A, p.(Asp532Asn): VCV000691957

c.1189G>A, p.(Ala397Thr): VCV000691958

c.207C>G, p.(Tyr69Ter): VCV000691955

\section{ACKNOWLEDGEMENTS}

We thank the patients and their families for participation in this study. We thank the Helmholtz-Zentrum Munich NGS core facility, especially Elisabeth Graf and as well as Gertrud Eckstein, Peter Lichtner, and Veronika Schwarzbauer for their excellent support. We also thank the Robert-Debré University Hospital for its full support. We also thank Fikret Yüzgülen for referring his patient. We thank Martin Krenn for his revision of the manuscript. This study was partly funded by Dietmar Hopp Stiftung (St. Leon-Rot, Germany) and the CNRG (Centre National de Génotypage (JFDeleuze).

\section{DISCLOSURE}

Y.C.S. is an employee of GeneDx, Inc. The other authors declare no conflicts of interest.

Publisher's note Springer Nature remains neutral with regard to jurisdictional claims in published maps and institutional affiliations.

\section{REFERENCES}

1. Lohmann K, Klein C. Next generation sequencing and the future of genetic diagnosis. Neurotherapeutics. 2014;11:699-707.

2. Ropers $\mathrm{HH}$. On the future of genetic risk assessment. J Community Genet. 2012;3:229-236.

3. Schule R, Wiethoff $S$, Martus $P$, et al. Hereditary spastic paraplegia: clinicogenetic lessons from 608 patients. Ann Neurol. 2016;79:646-658.

4. Zech $M$, Jech $R$, Wagner $M$, et al. Molecular diversity of combined and complex dystonia: insights from diagnostic exome sequencing. Neurogenetics. 2017; 18:195-205

5. Sonntag M, Blosa M, Schmidt S, et al. Synaptic coupling of inner ear sensory cells is controlled by brevican-based extracellular matrix baskets resembling perineuronal nets. BMC Biol. 2018;16:99.

6. Kwok JC, Dick G, Wang D, Fawcett JW. Extracellular matrix and perineuronal nets in CNS repair. Dev Neurobiol. 2011;71:1073-1089.

7. Probstmeier R, Nellen J, Gloor S, Wernig A, Pesheva P. Tenascin-R is expressed by Schwann cells in the peripheral nervous system. J Neurosci Res. 2001;64:70-78.

8. El Ayachi I, Fernandez C, Baeza N, De Paula AM, Pesheva P, FigarellaBranger D. Spatiotemporal distribution of tenascin- $R$ in the developing human cerebral cortex parallels neuronal migration. J Comp Neurol. 2011;519:2379-2389.

9. Woodworth A, Pesheva P, Fiete D, Baenziger JU. Neuronal-specific synthesis and glycosylation of tenascin-R. J Biol Chem. 2004;279:10413-10421.
10. Jakovcevski I, Miljkovic D, Schachner M, Andjus PR. Tenascins and inflammation in disorders of the nervous system. Amino Acids. 2013;44:1115-1127.

11. Weber $P$, Bartsch $U$, Rasband $M N$, et al. Mice deficient for tenascin-R display alterations of the extracellular matrix and decreased axonal conduction velocities in the CNS. J Neurosci. 1999;19:4245-4262.

12. Freitag $S$, Schachner $M$, Morellini F. Behavioral alterations in mice deficient for the extracellular matrix glycoprotein tenascin-R. Behav Brain Res. 2003;145:189-207.

13. Bruckner G, Grosche J, Schmidt S, et al. Postnatal development of perineuronal nets in wild-type mice and in a mutant deficient in tenascinR. J Comp Neurol. 2000;428:616-629.

14. Nikonenko A, Schmidt S, Skibo G, Bruckner G, Schachner M. Tenascin-Rdeficient mice show structural alterations of symmetric perisomatic synapses in the CA1 region of the hippocampus. J Comp Neurol. 2003:456:338-349.

15. Chiquet-Ehrismann R, Orend G, Chiquet M, Tucker RP, Midwood KS. Tenascins in stem cell niches. Matrix Biol. 2014;37:112-123.

16. Xu JC, Xiao MF, Jakovcevski I, et al. The extracellular matrix glycoprotein tenascin- $R$ regulates neurogenesis during development and in the adult dentate gyrus of mice. J Cell Sci. 2014;127(Pt 3):641-652.

17. Dufresne D, Hamdan FF, Rosenfeld JA, et al. Homozygous deletion of Tenascin- $R$ in a patient with intellectual disability. J Med Genet. 2012;49:451-454.

18. Lynch DS, Rodrigues Brandao de Paiva A, Zhang WJ, et al. Clinical and genetic characterization of leukoencephalopathies in adults. Brain. 2017;140:1204-1211

19. Hesse V, Schnabel O, Judis E, Cammann H, Hinkel J, Weissenborn J. Längsschnittstudie des aktuellen Wachstums 0- bis 6-jähriger deutscher Kinder: Teil 2. Monatsschr Kinderheilkd. 2016;164:892-912.

20. Sobreira N, Schiettecatte F, Valle D, Hamosh A. GeneMatcher: a matching tool for connecting investigators with an interest in the same gene. Hum Mutat. 2015;36:928-930.

21. Vriend G. WHAT IF: a molecular modeling and drug design program. J Mol Graph. 1990;8:52-56.

22. Krieger $E$, Koraimann G, Vriend $G$. Increasing the precision of comparative models with YASARA NOVA-a self-parameterizing force field. Proteins. 2002;47:393-402.

23. Jacobs SA, Diem MD, Luo J, et al. Design of novel FN3 domains with high stability by a consensus sequence approach. Protein Eng Des Sel. 2012:25:107-117.

24. Porebski BT, Nickson AA, Hoke DE, et al. Structural and dynamic properties that govern the stability of an engineered fibronectin type III domain. Protein Eng Des Sel. 2015;28:67-78.

25. Lundell A, Olin Al, Morgelin M, al-Karadaghi S, Aspberg A, Logan DT. Structural basis for interactions between tenascins and lectican C-type lectin domains: evidence for a crosslinking role for tenascins. Structure. 2004;12:1495-1506.

26. Lek M, Karczewski KJ, Minikel EV, et al. Analysis of protein-coding genetic variation in 60,706 humans. Nature. 2016;536:285-291.

27. Scott EM, Halees A, Itan Y, et al. Characterization of Greater Middle Eastern genetic variation for enhanced disease gene discovery. Nat Genet. 2016;48:1071-1076.

28. Fattahi Z, Beheshtian M, Mohseni M, et al. Iranome: a catalog of genomic variations in the Iranian population. Hum Mutat. 2019;40:1968-1984.

29. Fuss $B$, Wintergerst ES, Bartsch $U$, Schachner $M$. Molecular characterization and in situ mRNA localization of the neural recognition molecule J1-160/180: a modular structure similar to tenascin. J Cell Biol. 1993;120:1237-1249.

30. Anlar B, Gunel-Ozcan A. Tenascin-R: role in the central nervous system. Int J Biochem Cell Biol. 2012:44:1385-1389.

31. Gogolla N, Caroni P, Luthi A, Herry C. Perineuronal nets protect fear memories from erasure. Science. 2009;325:1258-1261.

32. Wen TH, Binder DK, Ethell IM, Razak KA. The perineuronal 'safety' net? Perineuronal net abnormalities in neurological disorders. Front $\mathrm{Mol}$ Neurosci. 2018;11:270.

33. Farlow JL, Robak LA, Hetrick $K$, et al. Whole-exome sequencing in familial Parkinson disease. JAMA Neurol. 2016;73:68-75.

34. Hawi Z, Yates $H$, Pinar A, et al. A case-control genome-wide association study of ADHD discovers a novel association with the tenascin R (TNR) gene. Transl Psychiatry. 2018;8:284. 